\title{
La privatización de la educación argentina: un largo proceso de expansión y naturalización
}

\author{
Susana E. Vior ${ }^{*}$, Laura R. Rodríguez ${ }^{* *}$
}

Resumen: En el artículo se revisan los momentos más importantes de la política educacional en Argentina en relación con el proceso de privatización de la vida social. Despejando algunas hipótesis habituales, se analiza ese proceso como resultado del modo en que se fueron resolviendo las demandas concretas de los grupos, fracciones o clases a lo largo de la historia nacional, culminando en la consolidación de un sólido sector de proveedores privados de educación, al amparo de medidas que le aseguran el financiamiento estatal y la presencia de sus intereses en la elaboración de la política educativa pública. Se interpreta el sentido del proceso en el marco del reacomodamiento de los intereses dominantes en esta etapa de recomposición capitalista, que realiza su hegemonía mediante la naturalización de las diversas formas de privatización de la educación nacional, tanto históricas como "novedosas", y oculta sus efectos concretos: la devaluación de la educación pública como bien universal y del papel del Estado como garante de su distribución igualitaria.

Palabras clave: privatización; política educativa; educación pública; Estado; tercera vía.

\section{Privatization of Argentinian education: an enduring process of expansion and naturalization}

Abstract: In this paper the most important moments of the educational policy in Argentine, related to the privatization process of social life, are reviewed. Putting aside some commonplace hypothesis, this process is analyzed as a consequence of the ways involved in the response to demands from classes or groups along the history of our country. This process led to the strengthening of a group of private suppliers of educational services, protected by measures that guarantee the financial support from the State and the presence of their interests when public education policies are prepared. The meaning of this process is considered within the frame of resetting the predominant forces in the present period of capitalism. Its hegemony is settled making the different forms of privatization -traditional or new of the national education system look natural, and hiding their real effects: the devaluation of public education as a universal value and of the role of the State to guarantee that education is equally distributed.

Key words: privatization; educational policy; public education; State; third way.

* Profesora Titular de Política Educacional Argentina y Comparada. Directora de la Maestría en Política y Gestión de la Educación, Universidad Nacional de Luján (UNLu). Argentina. svior@ unlu.edu.ar

** Docente del Departamento de Educación (UNLu). laura.rodriguez57@gmail.com 
La necesidad de analizar teórica y empíricamente las características del proceso de privatización más reciente, sufrido por la Argentina, y sus manifestaciones en la educación, nos obligó a revisar algunos momentos significativos en el desarrollo de nuestra política educacional. El presente trabajo anticipa una síntesis de la investigación en curso.

A partir de fines de los años'70, los sistemas educativos públicos fueron fuertemente cuestionados por un discurso que resaltó las bondades de los agentes privados y criticó la hipertrofia de un Estado ineficiente y deficitario. En ese momento tomó forma un proyecto global y una estrategia consistente de privatización, articulada con la expansión internacional de la ideología neoliberal. Llegada al poder con los regímenes conservadores de Thatcher y Reagan, luego de algo más de treinta años ha logrado naturalizar la mercantilización de la educación y fragmentar el espacio público en un conglomerado de intereses particulares. Este "nuevo" impulso privatizador de la educación pública es un fenómeno cuyas características esenciales están presentes en los países de la región alcanzados por las "recomendaciones" de los organismos internacionales, aunque con diferencias en su profundidad, alcance y modalidades. Esas diferencias son producto tanto de la historia de cada sistema, como de las condiciones sociales, políticas, culturales y económicas vigentes durante esta expansión reformadora, que revirtió los logros alcanzados en el Estado de Bienestar Keynesiano y sus parientes pobres, los "Estados Benefactores Latinoamericanos". En el caso argentino, la privatización actual es resultado de un proceso iniciado junto con la fundación del sistema educativo, pero cuyos avances fueron encubiertos, de modo tal que el tema fue sustrayéndose del debate público.

Una hipótesis ampliamente difundida para interpretar ese proceso ha sido, por ejemplo, vincular los avances del proceso privatizador con los períodos dictatoriales. Sin embargo, el análisis del desarrollo cuantitativo del sistema no permite la confirmación empírica de esa afirmación. En trabajos anteriores hemos planteado que, si bien los gobiernos de facto sustentaron proyectos educativos privatizadores coherentes con proyectos de privatización de toda la vida social (Tedesco et al, 1983; Oszlak, 1984), no siempre lograron aumento de la participación del sector en la provisión educativa. Por otra parte, muchos gobiernos constitucionales favorecieron medidas que apoyaron procesos privatizadores, aún en el marco de discursos defensores de la educación pública (Krawczyk et al, 1986). En realidad, la evolución de esos impulsos privatizadores y su relación con el desarrollo del sistema y de las políticas educativas parece ser fruto del modo en que se fueron resolviendo las demandas concretas de los grupos, fracciones o clases a lo largo de su historia. Este es el enfoque que sustenta nuestra exposición, que también requiere que explicitemos nuestro concepto de "lo público".

En nuestro marco analítico, la esencia de "lo público" reside en el reconocimiento de "asuntos de interés general" y "capacidades colectivas" que el Estado 
liberal debe garantizar para hacer realidad la promesa democrática (Iazzeta, 2008). Históricamente, en el Estado capitalista, la dicotomía público/privado es consustancial a la reivindicación secular del Estado liberal como garante de la universalidad de derechos ciudadanos frente a la fragmentación de intereses, en muchos casos contradictorios. En el caso de nuestro país, su momento fundacional es el de la organización definitiva de la Nación, instituida por la Constitución democráticoliberal de 1853.

\section{Etapas en el proceso de privatización educacional}

Si bien debe reconocerse la presencia de algunas condiciones preexistentes a la creación del Estado nacional (1853), en el proceso de privatización educativa entendido como retiro paulatino del Estado de su función educadora, identificamos cuatro etapas, vinculadas con distintas coyunturas de cada bloque histórico.

La educación privada como componente residual en el Estado Docente ( 853 1947)

Nuestra educación se constituyó como un sistema mixto, marcado por la oposición entre concepciones subsidiaristas, defensoras del papel de la familia y la Iglesia, y concepciones defensoras de la principalidad del Estado en educación. Esta disputa se expresó de diferentes maneras en la época del conservadurismo liberal (1853-1916), en la de los gobiernos radicales (1916-1930) y, luego de la crisis económica mundial de 1929, en la restauración conservadora facilitada por el primer golpe militar (1930-1945).

Durante la hegemonía del Estado liberal, éste se reservó un rol principal en la provisión y regulación del servicio educativo, garantizando la libertad de enseñar pero reservándose el otorgamiento de certificados y títulos y el contralor de las escuelas privadas, usualmente denominadas "escuelas particulares". Durante ese período se sentaron las bases y se expandió el sistema público de educación primaria gratuita, y secundaria y superior (universitaria y no universitaria) arancelada. Un pequeño segmento de educación no provista por el Estado estaba, fundamentalmente, en manos de la Iglesia católica y de diferentes colectividades de inmigrantes, libre o fiscalizada por el poder público y concentrada en las regiones más desarrolladas del país. Sus expresiones legales, según los principios establecidos por la Constitución de 1853, fueron la Ley 934 sancionada en 1878, "De libertad de enseñanza", la Ley 1420, de 1884, "De educación común”, y la Ley Avellaneda, de 1885, para la enseñanza universitaria. En el segmento gratuito de ese sistema educativo existió desde el inicio cierto grado de "privatización histórica" derivada del sistema de cooperadoras escolares que permitía recibir aportes voluntarios de las familias con fines asistenciales o dirigidos a mejoras de infraestructura y equipamiento que 
generó una primera diferenciación entre las escuelas públicas de acuerdo con las condiciones económicas de su población.

El esfuerzo educativo del Estado liberal enfrentó al avance de las fracciones conservadoras de la oligarquía y a las demandas orgánicas de la Iglesia como proveedora de educación ${ }^{1}$, sobre todo en la formación de maestros. El resultado de este enfrentamiento fue el crecimiento en el número de instituciones y de la matrícula privada, muy lento en el nivel primario y moderado en el nivel secundario. En 1930, por ejemplo, el 8,5\% de la matrícula de nivel primario era privada, mientras que este porcentaje ascendía al $22 \%$ en el nivel medio, con variaciones según la modalidad y con fuerte concentración en el magisterio (Tedesco, 1986). Se mantuvo el control de la enseñanza impartida por los establecimientos privados, obligados a someterse a la fiscalización estatal, a los efectos del otorgamiento de la acreditación de los estudiantes mediante exámenes ante tribunales mixtos (profesores oficiales y privados) ${ }^{2}$.

La consolidación de este proceso fue facilitada por la forma específica en que se reestructuró el nuevo bloque en el pasaje a la etapa de industrialización por sustitución de importaciones durante el primer peronismo. El ingreso a la participación política y social de nuevos grupos, obreros y trabajadores urbanos, empleados, funcionarios públicos y profesionales en un marco de expansión económica, tuvo como correlato un nuevo ciclo de expansión y diferenciación del sistema educativo. Y es en este período en el que observamos una alteración significativa en el papel jugado por el Estado en la educación: el establecimiento de un sistema de subsidios estatales a la educación provista por establecimientos particulares. Fruto del espacio de poder conquistado por el nacional-catolicismo en el golpe de Estado de 1943 constituyó, simultáneamente, una forma de ampliar la oferta educativa y cumplir compromisos adquiridos con la Iglesia. Se concretó a través de medidas tales como la incorporación por decreto de la enseñanza religiosa en las escuelas públicas (1943) -ratificada legalmente durante el primer gobierno del Gral. Perón (1946) - y, sobre todo, por la Ley 13.047, dictada en $1947^{3}$.

\section{La privatización subsidiada y la ampliación de la provisión privada de educación}

I En 1922 se creó el Consejo Superior de Educación Católica. Luego de diversas alternativas, en 1949, da origen al CONSUDEC, y crea un Instituto del Profesorado, reconocido por el Estado (Decreto 5933/49).

2 En 1936, el sector privado logró generar su propio espacio de conducción técnico-pedagógica dentro del aparato burocrático del Estado: la Inspección Técnica General de la Enseñanza Incorporada, exclusiva para los establecimientos privados. Ver: Vior y Misuraca (1998).

3 Simultáneamente, el Decreto 29.337/49 estableció la gratuidad del nivel universitario al suspender el cobro de aranceles con retroactividad a junio de ese año como adecuación a la nueva Constitución. El arancelamiento fue restablecido durante la dictadura del período 1976- 1983, medida que el gobierno de R. Alfonsín dejaría sin efecto, recuperando la gratuidad del nivel.

Pro-Posiçôes, Campinas, v. 23, n. 2 (68), p. 91-104, maio/ago. 2012 
durante el desarrollo y la primera crisis del Estado Benefactor Periférico (I 947- 1989)

La Ley 13.047/47 denominada "Estatuto del Docente Privado" inicia un nuevo período en el que la lógica del proceso de privatización se realiza, por un lado, a través de la consolidación del principio de subsidiariedad del Estado (establecido en la Constitución de 1949) y el desarrollo de un sistema de financiamiento público a las instituciones privadas; $\mathrm{y}$, por otro, a través de la legitimación social y política del papel de los proveedores privados de educación en todos los niveles educativos, con un deterioro paulatino del poder fiscalizador del Estado sobre sus acciones. La evolución de este proceso, sumada a la expansión de la matrícula pública sin un aumento correlativo de las inversiones necesarias, crea las condiciones para una marcada segmentación del sistema educativo ${ }^{4}$.

Por ejemplo, el subsidio público no los privaba de cobrar aranceles, aunque bajo un sistema de regulaciones y con la exigencia de mantener becas de estudio para al menos un $10 \%$ de alumnos da cada curso o grado. Sucesivas modificaciones de la legislación fueron reorganizando las categorías de establecimientos (Decreto 15/64, por ejemplo, durante el gobierno constitucional del Dr. A. Illia) y extendiendo los niveles alcanzados por el beneficio (Decreto 1621/69, incorporando a los jardines de infantes y escuelas diferenciales).

Para el contralor de los establecimientos, subsidiados y no subsidiados, bajo órbita federal fue creado un organismo específico dentro de la cartera educativa. A medida que fue produciéndose la transferencia del servicio educativo prestado por la Nación a las provincias, fueron creándose organismos provinciales similares. Desde su origen, estuvieron integrados por representantes de los intereses sectoriales de los establecimientos privados ${ }^{5}$ lo que, sumado a una serie de modificaciones de la normativa, facilitó la independencia progresiva de los controles estatales ${ }^{6}$. La autonomía creciente del sector privado fue acompañada -en el gobierno de

4 A partir de dicha ley, los establecimientos particulares pudieron ser financiados por fondos públicos, medida justificada como aporte complementario del Estado en el marco de la "justicia social". Junto a establecimientos privados "libres", encontramos entonces instituciones "adscriptas a la enseñanza oficial" que accedieron (en distintas proporciones establecidas por la reglamentación) al sostén económico del Estado para el pago de los salarios docentes.

5 Por ejemplo, la SNEP creada por el decreto 9247/60 contaba en su estructura con un Consejo Consultivo entre cuyos miembros debían encontrarse vocales designados por el PEN, a propuesta del Ministerio de Educación entre "especialistas que sean además figuras representativas de los intereses y orientaciones de la enseñanza privada o tengan adhesión a sus fines".

6 El régimen fue extendido llegando, a comienzos de los '70, a abarcar a establecimientos preprimarios, primarios, secundarios y terciarios no universitarios, de manera que todos estuvieron autorizados a "matricular, calificar, examinar, promover, otorgar pases, certificados y diplomas y a aplicar el régimen disciplinario y de asistencia de los alumnos, de acuerdo con las normas que dicte el Ministerio de Educación y Justicia" (Decreto 37//64, art.4). Simultáneamente, fueron relajándose las exigencias pedagógicas respecto del cumplimiento de los programas oficiales y la organización de la enseñanza.

Pro-Posiçôes, Campinas, v. 23, n. 2 (68), p. 91-104, maio/ago. 2012 
facto del Gral. Onganía- por un discurso que abandonó la diferenciación "oficial/ público" - "particular/privado" que había sido la original base de legitimación de la educación estatal (garante de los intereses comunes), para sustituirla por la distinción entre "público de gestión estatal", y "público de gestión privada", ocultando la propiedad de las instituciones y el status diferencial de sus docentes, tratados como "empleados de comercio" y no como funcionarios públicos.

En este marco general, la expansión de la matrícula privada siguió un patrón evolutivo más dinámico que el de la educación pública, caracterizado por un crecimiento lento (con historia y comportamientos particulares según el nivel educativo que se considere), influido por las características coyunturales de la economía nacional: estancamiento en períodos de retracción y ajuste, y recomposición en períodos de crecimiento ${ }^{7}$. Así, sobre una base de participación, en el año 1955 , de un $8,5 \%$ de la matrícula de la enseñanza primaria, un 18,5\% de la de secundaria, y un $6,2 \%$ de la terciaria no universitaria, asistimos a la consolidación del segmento de educación privada, que en 1988 llega al 18\% de la matrícula primaria, al $28,7 \%$ de la secundaria, y al $27,6 \%$ de la terciaria no universitaria (Fernández et al, 1997) ${ }^{8}$.

Paralelamente, los defensores de la privatización lograron en esta etapa quebrar el monopolio estatal de la educación universitaria a partir del Decreto 6403/55, que estableció la posibilidad de creación de universidades privadas. La reglamentación del decreto se realizó recién durante el gobierno de A. Frondizi, a través de la Ley $14.557 / 58$ y su Decreto Reglamentario 1404/59, que especificó que las universidades privadas no podrían recibir "recursos estatales" (Art. $1^{\circ}$ ). Durante el gobierno del Gral. Onganía, la Ley 17.604/67 reglamentó en forma más integral la actividad de las universidades privadas, y se creó el Consejo de Rectores de Universidades Privadas (CRUP). Entre 1958 y 1963, el sector privado -sobre todo el ligado a la Iglesia Católica- abrió más universidades ${ }^{9}$ que las creadas por el Estado desde $1853^{10}$. Inicialmente, los títulos otorgados por ellas debían ser validados a través de exámenes habilitantes para ser considerados equivalentes a los otorgados por las

7 Entre 1955 y 1980, las tasas de crecimiento de la matrícula privada primaria y terciaria no universitaria fueron hasta tres veces más altas que las tasas de crecimiento registradas en el sector público. En el nivel medio encontramos este mismo fenómeno, un poco menos marcado y con un estancamiento del sector privado a partir de 1970. Ver: Fernández et al (1997, p. I I-29).

8 En el caso particular de la formación de maestros, en 1969, por ejemplo, la matrícula de la enseñanza media normal en el segmento privado alcanzaba ya al 52\% del total. Vior y Misuraca (1998, p. 25).

9 Se crearon 8 universidades: Universidad Católica de Córdoba, Católica de Santa Fe, Católica de Cuyo, Universidad del Salvador, Católica Argentina, del Museo Social Argentino, de Mendoza, y Universidad J. A. Maza.

10 Entre 1853 y 1963, el Estado (nacional o provincial) creó las universidades de: La Plata, Tucumán, Litoral, Tecnológica (sobre la base de la Universidad Obrera Nacional), Nordeste y Sur. Sumadas a la Universidad de Córdoba y la de Buenos Aires, llegaban, en 1963, a 8 establecimientos.

Pro-Posições, Campinas, v. 23, n. 2 (68), p. 91-104, maio/ago. 2012 
universidades nacionales. Supervisadas laxamente por el Ministerio de Educación, sostuvieron en forma continuada la defensa de sus intereses sectoriales a través del CRUP, mientras las universidades públicas experimentaban intervenciones y regulaciones cambiantes que interrumpieron en forma intermitente las experiencias de coordinación autónoma entre las instituciones y que, sobre el final del período, serían arrasadas por la última dictadura militar.

Llegados los años '90, la educación privada se había expandido en forma sostenida, a pesar de estancamientos coyunturales provocados por el impacto de las políticas económicas, en el desarrollo de los sectores medios, principales beneficiarios de la privatización educativa subsidiada por el Estado. Mediante la capitalización diferencial derivada del subsidio público otorgado con criterios cada vez menos alineados con el objetivo declamado (complementar el esfuerzo público dirigido a democratizar el servicio educativo), de la posibilidad de cobrar aranceles en forma cada vez más discrecional y del incumplimiento del régimen de becas, en esta etapa quedó constituido un sólido sector de proveedores privados de educación, cuya situación contrasta cada vez más con la desprotección creciente de la escuela pública, sometida a los vaivenes de gestiones autoritarias $\mathrm{y}$ crisis fiscales.

\section{La privatización "en" la educación en el Estado Periférico Neoconservador Neoliberal (1990-2003)}

A esta herencia de privatización, cuyos impulsos esenciales habían provenido de las fracciones conservadoras anti-liberales de la sociedad, se suman en este periodo nuevas formas surgidas del dominio mundial del nuevo conservadurismo de mercado, que logra afectar al núcleo mismo de la función educadora en el ámbito estatal y a la política educativa pública. El análisis realizado por Ball y Youdell sobre los procesos de privatización educativa a nivel mundial aporta categorías fértiles para interpretar el caso argentino. Ellos los caracterizan como: a) formas de privatización endógena "en" la educación pública-que "implican la importación de ideas, métodos y prácticas del sector privado a fin de hacer que el sector público sea cada vez más como una empresa y crecientemente comercial"- y b) formas de privatización exógenas "de" la educación pública -que "implican la apertura de los servicios de educación pública a la participación del sector privado a través de modalidades basadas en el beneficio económico, y la utilización del sector privado en cuanto a la concepción, la gestión o la provisión de diferentes aspectos de la educación pública”- (Ball; Youdell, 2007, p. 8-9).

Resulta claro que, en Argentina, el embate de Nueva Derecha sobre la educación pública se realizó sobre un sistema educativo que ya había sufrido un proceso de privatización importante. La sanción de la Ley Federal de Educación (1993) le 
otorgó respaldo legal definitivo ${ }^{11}$. Ya sea a través de políticas activas o por medio de "no-políticas", el Estado consolidó el sustrato "histórico" de privatización, garantizando condiciones de financiamiento diferenciales que aseguraron la "rentabilidad" y la calidad diferencial de un segmento privado subsidiado, que reforzaron la segmentación del sistema educativo según condición socio-económica de los alumnos.

La "Reforma Educativa" de los '90 invistió al Estado subsidiario argentino de nuevas funciones: el Estado subsidiario evaluador, difusor del paradigma de la Nueva Gestión Pública, divulgó concepciones empresariales que apelaron a la calidad y a la excelencia, a la visión estratégica y a la rendición de cuentas, concepciones mercantilizadas de la política y la administración educativa basadas en los incentivos y la competencia, una verdadera privatización de las identidades educativas que asimiló estudiantes con clientes y educadores con empleados evaluados según su "eficiencia y rendimiento", directivos con gerentes, e instituciones educacionales con empresas en cuasi mercados. Los efectos se manifestaron con claridad en la tercerización de los comedores escolares, los servicios de transporte y de limpieza de los establecimientos, servicios de orientación educacional, en la sustitución del Estado como agente principal de la formación de los docentes en servicio (Red Federal de Formación Docente Continua, 1993) o la experiencia de escuelas charter y de bonos educativos de la Provincia de San Luis (Quiroga, 2009). En el nivel universitario, si bien pudieron frenarse las pretensiones de anular la gratuidad de los estudios de grado, se generalizó el cobro de aranceles en los posgrados y otros servicios educativos brindados por las instituciones, se implantó la lógica empresarial en la gestión y gobierno y se generalizó el uso de mecanismos competitivos para el reparto de recursos públicos (FOMEC, Programas Especiales de Financiamiento bajo el control del Ministerio de Educación, etc.)

Al haberse transferido, en 1992, los servicios educativos de todos los niveles (menos el universitario), a las jurisdicciones provinciales, son ahora las provincias (y la Ciudad Autónoma de Buenos Aires) las que administran la lógica regresiva de la política de financiamiento subsidiado, beneficiando a los sectores socioeconómicos de mayores recursos, en el marco de una distribución cada vez más desigual de la riqueza. Con algún estancamiento en el período de crisis 1999-2002, la educación privada se consolidó. Por su parte, en 1999, el 15\% del gasto público total en educación se destinaba a escuelas privadas, con algunas diferencias entre jurisdicciones. La participación de la matrícula universitaria privada se mantuvo

II Su art. 4 es claramente de inspiración neoconservadora: "Las acciones educativas son responsabilidad de la familia, como agente natural y primario de la educación, del Estado nacional como responsable principal, de las provincias, los municipios, la Iglesia Católica, las demás confesiones religiosas oficialmente reconocidas y las organizaciones sociales", que se constituyen, junto a las "instituciones educativas estatales", como "entidades de gestión privadas reconocidas" (art. 7) que mantienen su normativa histórica (Capítulo V, arts. 36 a 38). 
estable a lo largo de los '90, en alrededor del 15\%. La escuela pública, que durante la mayor parte del siglo XX había sido un espacio de encuentro de sectores populares y medios, fue volviéndose más homogénea en términos de los grupos sociales atendidos, quedando como el espacio educativo de los "pobres", cada vez más distante de las condiciones materiales en las que se enseña y aprende en los establecimientos privados.

\section{A partir de 2003: privatización "de" la política educativa}

La crisis de 2001 dio lugar a una recomposición del bloque de fuerzas dominante en los '90 y al ascenso al poder de gobiernos que, definiéndose como progresistas, han desarrollado un discurso crítico del neoliberalismo y de su reforma educativa.

En la política desplegada a partir de 2003 pueden reconocerse, sin embargo, varias continuidades con la etapa anterior. Desde nuestro punto de vista, son consecuencia lógica del programa político que la sustenta: el de la socialdemocracia de Tercera Vía. En lo que se refiere al proceso de privatización creciente de la educación, se caracteriza por incorporar una nueva dimensión: la privatización de la política educativa.

Si en el período precedente, los intereses corporativos de los agentes privados se materializaron en su participación directa en los organismos estatales de fiscalización de la educación primaria, secundaria y superior no universitaria privada, en esta etapa, las reformas implican su ingreso en todos los organismos de elaboración de políticas y de gobierno y coordinación de la educación en general, en pie de igualdad con los funcionarios gubernamentales que tradicionalmente habían representado el "interés público". De esta manera, se intensifica la intervención de grupos, sectores e instituciones privadas en las instancias encargadas del seguimiento y la evaluación del sistema, y en los espacios ministeriales de asesoramiento y consulta. Simultáneamente, se limita la participación de docentes, estudiantes y padres en el gobierno del sistema y en las instituciones educativas, reduciéndolo a la colaboración en el logro de objetivos de proyectos institucionales en cuya definición no participaron (Más Rocha y Vior, 2009). Bajo la lógica de la recentralización neoconservadora (descentralizar responsabilidades centralizando las decisiones esenciales) esta presencia es refrendada por la Ley de Educación Nacional de 2006, acompañada por un discurso crítico de la Ley Federal de Educación de $1993^{12}$.

12 Los cuerpos representativos de la educación privada participan, según el art. 66 de la LEN, en el Consejo Consultivo de Políticas Educativas del Consejo Federal de Educación, en el Consejo de Universidades a través del Comité Ejecutivo del CRUP, tienen I representante en la CONEAU y los arts. 44 y 45 de la LES los habilitan para crear entidades privadas de evaluación y acreditación (aunque con reconocimiento previo del MEd, dictamen favorable de la CONEAU, y aceptación de los patrones y estándares oficiales). Los intereses particulares están representados además por la incorporación, en dos de los Consejos Consultivos del Consejo Federal de Educación, 


\section{La naturalización de la privatización de la educación en un contexto de Tercera Vía}

A pesar de la recuperación del discurso democrático centrado en la igualdad y en la ciudadanía, las medidas no se dirigen a revertir el carácter regresivo e injusto de la privatización, un factor más que contribuye al deterioro de la educación pública, verdadera "privatización por desgaste" (Gamallo, 2008). Recientes investigaciones (Rivas, 2010; Mezzadra; Rivas, 2010) evidencian que la normativa y los procesos de asignación de subsidios al sector privado son discrecionales, y se dirigen en alta proporción a establecimientos que no atienden a los sectores sociales pobres y que benefician, mayoritariamente, a escuelas religiosas.

Los poderes públicos no proporcionan información sobre los criterios de asignación de recursos, no se controla el grado de cumplimiento de la obligación de otorgar becas y se ha desregulado crecientemente el cobro de aranceles adicionales bajo la justificación de compensar los "mayores costos" derivados de los aumentos salariales a los docentes. Por ejemplo, en la Ciudad Autónoma de Buenos Aires, los topes máximos arancelarios establecidos no se corresponden con el porcentaje de aporte que reciben las escuelas, y se autoriza el cobro de aranceles muy superiores por diversos adicionales ${ }^{13}$ que distorsionan seriamente la finalidad del aporte estatal (Sigal, 2010), llegando en algunos casos a habilitar a los establecimientos a duplicar el subsidio del Estado y a que "escuelas que cobran las cuotas más altas dentro de un nivel educativo y modalidad lleguen a cobrar hasta un $366 \%$ más que las escuelas que cobran el arancel más bajo; y sin embargo, el aporte estatal previsto es sólo un $60 \%$ más alto" (ibíd.: 14). Se explica así que entre 2005 y 2009, el gasto público en subsidios a la educación privada haya elevado su participación en el total del gasto educativo del 15\% al 18,4\% (ibíd.: 19). Por otro lado, la provincia de Córdoba destinaba a establecimientos privados, en 2006, el 24\% de su presupuesto educativo, seguida por Santa Fe (20\%), Misiones (16\%), Entre Ríos (15\%), y Buenos Aires (14\%).

Paralelamente, el segmento privado de proveedores de educación sigue aumentando. Según datos del 2006, el 27\% de los alumnos de todos los niveles educativos del país (excepto el universitario) concurrían a establecimientos privados; las diferencias entre provincias muestran que las jurisdicciones más pobres son

de representantes de organizaciones sociales y no gubernamentales vinculadas con la educación (Res. CFE N ${ }^{\circ}$ 6/07) sin explicitar cuáles son consideradas como tales ni con qué criterios se los elige, mientras se incrementa la participación de organizaciones empresariales.

13 Aún recibiendo el 100\% del aporte estatal, la Disposición 300/DGEGP/2009 estableció la posibilidad de las escuelas de percibir aranceles adicionales en concepto de cuotas adicionales de mantenimiento y equipamiento, transporte, comedor escolar, seguros de vida o servicios de emergencias médicas y cuotas por Asociaciones de Padres, sin contar los habituales aranceles "extra programáticos" justificados por la incorporación de materias o actividades no contempladas por el núcleo curricular oficial obligatorio. 
precisamente las menos "privatizadas": Chaco y Formosa sólo tienen un 9\% de la matrícula en establecimientos privados, frente a la Ciudad de Buenos Aires (49\%), provincia de Buenos Aires (33\%), Córdoba (32\%) y Santa Fe (30\%) (Mezzadra y Rivas, 2010) ${ }^{14}$. Por su parte, y de acuerdo con datos de 2009 , ya el $20 \%$ de los alumnos de nivel universitario concurría a instituciones privadas (SPU, 2010).

El discurso político educativo (y en general de las políticas sociales) del pensamiento neoliberal-neoconservador triunfante en los '90 se ha vuelto de sentido común en el nuevo siglo, en virtud de una "confluencia perversa" entre dos versiones políticas que coinciden en la demanda de una sociedad civil activa: el proyecto neoliberal (que reclamó "más sociedad y menos Estado") y el proyecto de la Tercera Vía (que reclama "más participación y solidaridad") (Dagnino, 2004). Se trata de categorías que legitiman medidas de política pública educativa atribuyéndoles carácter democrático, popular y progresista, en virtud de su capacidad para hacer referencia a ciertos ideales de tradición socialdemócrata. Su sencilla inclusión evita un análisis riguroso de su contenido, implementación y resultados concretos. Dado su carácter ambiguo, fueron incorporados fácilmente al discurso político nacional de gobernantes, funcionarios, académicos y dirigentes pertenecientes a diferentes extracciones políticas: "equidad", "participación”, "democratización”, "desarrollo sostenible", "inclusión", "concertación”, o "consenso". Constituyen reinterpretaciones encubridoras de los viejos problemas que siguen sin resolverse: igualdad, participación y democracia. La LEN, por ejemplo, mantiene la distinción entre gestión estatal y gestión privada de la educación pública, agregando las de "gestión social y cooperativa”. Estas nuevas instituciones, aparentemente diferentes de las escuelas estatales y privadas, serían las encargadas de dar respuesta a las demandas de la sociedad civil, que pasa a ser "la única institución portadora de virtudes políticas, e inclusive por parecer 'no política', valorizada como si se tratara de un nuevo agente de transformación histórica y expresión de los deseos libertarios y de justicia social” (Sorj, 2005, p. 65).

El concepto gramsciano de "sociedad civil", entendido como espacio de la lucha de clases y de proyectos sociales contrastantes, es neutralizado asimilándolo a un "tercer sector" más allá del Estado y del mercado ${ }^{15}$, caracterizado por respuestas

I4 Según datos del Ministerio de Educación para 2010 la matrícula privada de la educación común en todas sus modalidades llegó al 28\%, discriminada como sigue: 33\% en el nivel inicial, 25\% en el nivel primario, 28\% en el nivel secundario, y $42 \%$ en el nivel terciario (DINIECE, Anuario 2010)

I5 En Argentina, uno de los factores que facilitó el abandono de la idea de un Estado garante de las conquistas democráticas y la preferencia por una "sociedad civil" abstraída de las confrontaciones de clase fue la evolución del pensamiento de la izquierda que, hacia fines de los '80, asimiló en bloque al Estado con su expresión autoritaria en los regímenes militares, a la vez que desfiguró el concepto gramsciano de "sociedad civil". Ver, por ejemplo: Portantiero, J. Carlos ( I 989). La múltiple transformación del Estado latinoamericano. Nueva Sociedad, Buenos Aires, No 104 , noviembre-diciembre 1989, p. 88-94. 
solidarias, cooperativas, de voluntariado, en el que las demandas sociales quedan reducidas al nivel corporativo de los intereses particulares. Esto se realiza como parte de una reconfiguración más amplia del Estado y sus modalidades de acción que no son una simple estrategia de transferencia de competencias y desregulación, asociada a supuestas tendencias para su re-democratización, sino un mecanismo por el que se consuma el abandono de la defensa de intereses comunes a través de políticas universales para una sociedad entendida como "nación".

El reacomodamiento de los intereses dominantes, en esta etapa de recomposición capitalista, realiza su hegemonía mediante un proceso de naturalización de las diversas formas de privatización de la educación nacional tanto históricas como nuevas. Sus efectos concretos son la devaluación de la educación pública como bien universal y del papel del Estado como garante de su distribución igualitaria; efectos antidemocráticos encubiertos por un discurso progresista de recuperación del Estado "para todos" que evita abordar seriamente el deterioro de la educación pública que atiende a los más pobres, y ofrece garantías crecientes para el desarrollo de la educación privada que atiende a los sectores de mayores ingresos. De esta manera, se hace retroceder las conquistas liberales relativas a las libertades, derechos y garantías de la democracia formal, así como los logros alcanzados por los sectores subordinados a lo largo del siglo XX.

No se puede olvidar que existe una tensión esencial en las democracias capitalistas, "donde lo sustantivo es el capitalismo y lo adjetivo es la democracia" (Borón, 2003). Reconociendo este límite, siguen siendo condiciones necesarias para la construcción de una democracia en la que el Estado, en tanto lugar donde se condensan relaciones de fuerza como dominio y hegemonía, es crucial como garante de esas conquistas, de manera que, aun "burgués", sigue siendo "público". Como la realización efectiva para todos es históricamente el fruto de conquistas de la acción colectiva, es necesario defender aquellas instancias del Estado capitalista que "expresan, aún contradictoriamente, los intereses de las clases subalternas. Se trata de redefinir esas instancias, volverlas a dotar de su sentido original desvirtuado en su expresión material concreta" (Castillo; Thwaites Rey, 1999, p. 90).

\section{Referencias bibliográficas}

BALL, S.; YOUDELL, D. Ly: Privatización encubierta en la educación pública. Informe preliminar. En: V CONGRESO MUNDIAL, jul. 2007, Bruselas: Internacional de la Educación.

BORÓN, A. La sociedad civil después del diluvio neoliberal. En: SADER, E.; GENTILI, P. (Comp.) La trama del neoliberalismo: mercado, crisis y exclusión social. Buenos Aires: CLACSO, 2003. Disponible en: http://bibliotecavirtual.clacso.org.ar/ar/libros/trama/ boron.rtf. Acceso en: mar. 2005. 
CASTILLO, J.; THWAITES REY, M. La vigencia del Manifiesto Comunista: su importancia para pensar el Estado y la democracia. En: BORÓN, A. Teoría y filosofía política, la tradición clásica y las nuevas fronteras. Buenos Aires: CLACSO, 1999. p. 77-90.

DAGNINO, E. ¿¿Sociedade civil, participação e cidadania: de que estamos falando? En: MATO, D. (Coord.) Políticas de ciudadanía y sociedad civil en tiempos de globalización. Caracas: FACES, Universidad Central de Venezuela, 95-110, 2004.

FERNANDEZ, Ma. A.; LEMOS, Ma. L.; WIÑAR, D. La Argentina fragmentada. El caso de la educación. Buenos Aires: IICE; Miño y Dávila, 1997.

GAMALLO, G. Variaciones en el acceso a la educación de gestión estataly privada en Argentina (1997-2006). Buenos Aires: Centro de Estudios en Políticas Públicas (CEPP), Documento de Trabajo n. 2, feb. 2008.

IAZZETTA, O. Lo público, lo estatal y la democracia. Iconos. Revista de Ciencias Sociales, Buenos Aires, n. 32, 49-60, 2008.

KRAWCZYK, N.; MALAJOVICH, A. M.; VIOR, S. Aportes para una desmitificación. Revista Argentina de Educación, Buenos Aires, año 4, n. 6, 7-16, oct. 1985.

MÁS ROCHA, S.; VIOR, S. Nueva legislación educacional: ¿̨nueva política? En: VIOR, S.; MISURACA, Ma. R.; MÁS ROCHA, S. (Comp.) Formación de docentes ¿qué cambió después de los '90 en las politicas y los currículos y las instituciones? Buenos Aires: Jorge Baudino, 17-46, 2009.

MEZZADRA, F.; RIVAS, A. Aportes estatales a la educación de gestión privada en la Provincia de Buenos Aires. Buenos Aires: CIPPEC. Programa de Educación. Área de Desarrollo Social, Documento de Trabajo n. 51, nov. 2010.

OSZLAK, O. Privatización autoritaria y recreación de la escena pública. Crítica y Utopía, Buenos Aires, n. 10-11, 1984.

QUIROGA, M. El emporio educativo. El aporte estatal subsidiario de equidad o bono educativo como dispositivo de fomento de la educación privada en San Luis. KAIROS. Revista de Temas Sociales. Proyecto Culturas Juveniles Urbanas. Universidad Nacional de San Luis, año 13, n. 23, abr. 2009. Disponible (versión digital) en: www.revistakairos. org. Acceso en: ago. 2010.

RIVAS, A. La ruta hacia la justicia y la transparencia de los aportes estatales a la educación de gestión privada. Buenos Aires: CIPPEC. Programa de Educación. Área de Desarrollo Social. Documento de Políticas Públicas — Recomendación n. 86, nov.2010.

SIGAL, M. (Dir.). Subsidios estatales a las escuelas de gestión privada en la Ciudad de Buenos Aires. Falta de transparencia y profundización de desigualdades educativas. Buenos Aires: Asociación Civil por la Igualdad y la Justicia (ACIJ); Fundación Tinker (TFI), 2010.

SORJ, B. La democracia inesperada. Buenos Aires: Prometeo, 2005.

TEDESCO, J. C.; BRASLAVSKY, C.; CARCIOFI, R. El proyecto educativo autoritario. Buenos Aires: FLACSO, 1983. 
TEDESCO, J. C. Educación y sociedad en la Argentina (1880-1945). Buenos Aires: Solar, 1986.

VIOR, S. (Dir.). Estado y educación en las provincias. Buenos Aires: Miño y Dávila, 1999.

VIOR, S.; MISURACA, Ma. R. Conservadurismo y formación de maestros. Revista Argentina de Educación, Buenos Aires, año 16, n. 25, 7-25, 1998.

Recebido em 05 de janeiro de 2012 e aprovado em 06 de março de 2012. 